\title{
ON THE EFFICIENCY OF THE SPLITTING AND ROULETTE APPROACH FOR SENSITIVITY ANALYSIS
}

\author{
Viatcheslav B. Melas \\ Department of Mathematics and Mechanics \\ St.-Petersburg State University \\ Bibliotechnaya sq. 2, Petrodvoretz, St. Petersburg, 198904, RUSSIA
}

\begin{abstract}
The paper is devoted to a brief review of a mathematical theory for the branching variance-reduction technique. The branching technique is an extension of von Neumann's splitting and Russian roulette approach. The efficiency increase is attained by parallel simulation of several independent paths of the random process under consideration, the number of which is regulated by procedures of splitting and roulette. These procedures are governed by a probability measure whose optimal choice is the main problem of the corresponding mathematical theory. The relation between splitting and roulette approach and sensitivity analysis is discussed in the introduction.
\end{abstract}

\section{INTRODUCTION}

Sensitivity analysis is a relatively new but substantially important approach. Its purpose consists of the estimation of simulation results uncertainty caused by various sources. These are (see, for example, Cheng, Holland, 1995) errors due to the stochastic nature of simulation, uncertainty of a given data used for the simulation model building, uncertainty of input information as well as simplifying assumptions about distributions of values included in the simulation model under consideration. However sensitivity analysis can be directed not only to the study of ready made models but also to the comparison of underlying simulation techniques from the point of view of uncertainty of results. Thus we can consider variance reduction techniques as a part of sensitivity analysis.

Stochastic simulation of complex systems of different kinds is based on computer reproduction of paths of the random process which describes the dynamic behavior of the system under consideration. However, direct-path simulation often is not efficient, because it demands too large a number of paths to attain sufficient precision, especially when evaluating rare-event probabilities. Techniques to increase computer simu- lation efficiency have been called variance reduction techniques. Reviews of such techniques can be found in Glynn, Iglehart (1988) and in Bratley, Fox, Schrage (1987). However, as a rule, these methods demand significant a priori information about the investigated system, for example, specification of Markov chain transition probabilities in analytical form or analytical computation of characteristics of random values (expectation and variance) which are strongly correlated with the system parameters of interest. If we have only an algorithmic description of the system then such information is usually inaccessible.

A quite universal technique, free of the disadvantage indicated above, was proposed by von Neumann under the name "splitting and Russian roulette technique," see Kahn (1956). The basis of this technique is parallel simulation of several paths of a random process. A procedure for splitting and a procedure for roulette is applied to each of these paths. These procedures are chosen depending on the significance of the information on the system parameters contained in the path. The efficiency of this technique depends on the algorithm for selecting the number of paths to which the current path is split, and on the algorithm for computing the cancellation probability for the current path. For particular cases the problem of optimal choice of such an algorithm was investigated by Glasserman et al. (1996).

A mathematical statement of the problem, based on the introduction of a probability measure which governs the processes of splitting and roulette, had been proposed and investigated in Melas $(1993,1994)$ and Ermakov, Melas (1995). The approach, corresponding to the problem statement, has been called the branching technique. This technique permits us to develop a mathematical theory, which has some similarity to the classical theory of regression experimental design. (The basis for this theory was established in Kiefer and Wolfowitz, 1959). Selecting the probability measure can be considered as part of the design of the simulation experiment. This approach 
permits us to find the experimental design that minimizes the sum of the number of steps for all the paths that are simulated, under the condition that a specified accuracy for the estimates is attained. These designs can be developed on the basis of current results of the simulation, and their efficiency can be investigated analytically or on the basis of simulation.

Note that the mathematical theory of the branching technique is investigated for the case of Markovchain simulation. However, this case seems to be of great importance since many real problems can usually be reduced to Markov-chain simulation (see, f.e., Sigman, 1988).

In the present paper a review of mathematical results, obtained in Melas $(1993,1994)$ and Ermakov, Melas (1995), is given. It is a developed and revised version of author's work (Melas, 1995). It is also shown here that the computation required by the branching method can be significantly less than the computation required by the direct simulation.

\section{STATEMENT OF THE PROBLEM}

We will consider the estimation of one or several linear functionals of the stationary distribution of a general homogeneous Markov chain, and, in particular, finite Markov chains, by means of computer simulation. In the present section we will state the assumptions which are necessary for the strict mathematical statement of the problem, and will present a description of the branching technique.

\subsection{Assumptions}

To begin, let us consider general Markov chains. Let $\left\{X_{n}\right\}$ be a Markov chain with state space $\Omega$, with transition probability function $P(x, d x)$ and with stationary distribution $\pi(d x)$. Let random variables $X_{n}$, $n=0,1, \ldots$ be defined on the same probability space. Let us denote by $X$ the set of possible values of these variables and by $A$ a $\sigma$-algebra of its subsets. Let the $\sigma$-algebra $A$ be countably generated and let $h_{i}(x)$, $i=1,2, \ldots, l$ be measurable functions on $(X, A)$. Let $\mu$ be a (nonnegative) measure on $(X, A)$.

Suppose, that

(a) $P(x, B)$ is a probability measure on $(X, A)$ for a fixed $x$, and a measurable function of $x$ for a fixed $B$,

$$
\int P(x, X) \mu(d x)=1
$$

(b) The chain $\left\{X_{n}\right\}$ is $\mu$-nonreducible, i.e., there exists a function $s(x)$ on $\mathrm{X}$ and a probability measure $\nu(B)$ such that

$$
P(x, B) \geq s(x) \nu(B)
$$

for any $x \in X, B \in A$,

$$
\int_{X} s(x) \mu(d x)>0
$$

(c) $h_{i}(x) \geq 0$,

$\int_{X} h_{i}(x) \pi(d x)<\infty$

$\int h_{i}(x) E\left\{\sum_{n=1}^{\tau(B)} h\left(X_{n}\right) \mid X_{0}=x\right\} \pi(d x)<\infty$,

for $i=1,2, \ldots, l, B \in A$ where $\tau(B)=\min \{n$ : $\left.X_{n} \in B\right\}$.

Without loss of generality $s(x)$ can be considered (see Nummelin, 1984) as an indicator of a set $S$, i.e.,

$$
s(x)= \begin{cases}1, & x \in S \\ 0, & \text { otherwise }\end{cases}
$$

For the case when $\left\{X_{n}\right\}$ is a homogeneous Markov chain with a finite number of states, $\Omega=$ $\{0,1, \ldots, m\}$, denote the transition matrix by $P$, and the stationary probability vector by $\pi=\left(\pi_{0}, \ldots, \pi_{m}\right)$.

Let us suppose, that

(d) $\exists n$ such that $\left(P^{n}\right)_{i j}>0$, where $\left(P^{n}\right)_{i j}>0$ is an element with subscripts $i, j$ of the matrix $P^{n}, i, j=0,1, \ldots, m$;

(e) $h_{i}(x)<\infty$, for $x \in \Omega, i=1,2, \ldots, l$, and $h_{i}(x) \geq 0$.

Condition (d) means that the chain is an aperiodic and positive recurrent chain.

\subsection{Simulation Problem}

It is required to estimate one or several functionals of the form

$$
J_{i}=\int_{X} h_{i}(x) \pi(d x), \quad i=1,2, \ldots, l
$$

with respect to simulation results.

In case of chains with finite state space these functionals have the form

$$
J_{i}=\sum_{x=0}^{m} h_{i}(x) \pi_{x}, \quad i=1,2, \ldots, l .
$$

\subsection{Branching Technique}

Let $\left\{X_{n}\right\}$ be a Markov chain of the general form described in $\S 1.1$. Let us introduce independent random variables $Z_{n}, n=0,1, \ldots$, where $P\left\{Z_{n}=1\right\}=$ $s\left(X_{n}\right), \quad P\left\{Z_{n}=0\right\}=1-s\left(X_{n}\right)$.

Let $\eta_{n}$ chains $\left\{X_{n}, Z_{n}\right\}$, denoted $\left\{X_{n}^{(\gamma)}, Z_{n}^{(\gamma)} ; \gamma=1,2, \ldots, \eta_{n}\right\}$, be simulated 
at the $n$-th step. A procedure for regulating $\eta_{n}$ can be introduced in the following way.

Definition. A measurable function $\beta(x)$ on $(X, A)$ with the following properties:

1) $\beta(x)=$ const $\quad x \in S$,

2) $\beta(x) \geq 0, \quad x \in \Omega$,

3) $\int_{B} \beta(x) \pi(d x)>0$, if $\pi(B)>0$

is called a branching density.

For finite Markov chains satisfying condition (d), conditions 2) and 3 ) are replaced by $\beta(x)>0, x \in \Omega$.

Introduce the random variable

$$
r(x, y)=\lfloor\beta(y) / \beta(x)\rfloor,
$$

with probability $1-\overline{\beta(y) / \beta(x)}$

and

$$
\begin{gathered}
r(x, y)=\lfloor\beta(y) / \beta(x)\rfloor+1, \\
\text { with probability } \frac{\beta(y) / \beta(x),}{\beta(x)}
\end{gathered}
$$

where $\lfloor a\rfloor$, with $a=\beta(y) / \beta(x)$, means the integer part of $a$ and $\bar{a}$ means the whole part of $a$.

At step zero, set $\eta_{0}=1$. At each following step $(n+1=1,2, \ldots)$ if $Z_{n+1}^{\gamma}=1$, then the simulation of path $\gamma$ discontinues. If $Z_{n+1}^{\gamma}=0$ and $X_{n}^{\gamma}=x$, $X_{n+1}^{\gamma}=y$, then when $\beta(y) / \beta(x)<1$ the simulation of path $\gamma$ discontinues with probability $1-\beta(y) / \beta(x)$. When $\beta(y) / \beta(x) \geq 1$ we simulate $r(x, y)-1$ additional paths, beginning from point $x$, where $k$-steps of these chains will be denoted by index $n+k$.

It can be shown (see Theorem 1 below), that the number $N=\left\{n: \eta_{k}>0, k<n, \eta_{n}=0\right\}$ is finite with probability 1 , and moreover $T_{\beta}=\sum_{n=0}^{N} \eta_{n}<$ $\infty$ with probability equal to 1 . Thus the simulation terminates with probability 1 . This process will be called a loop.

Let us simulate $k$ loops: $\left\{X_{n}^{\gamma}(i) ; \quad \gamma=\right.$ $\left.1,2, \ldots, \eta_{n}(i)\right\}, \quad i=1,2, \ldots, k$. Let us determine estimators of the functionals $J_{j} \quad(j=1,2, \ldots, l)$ by the formula

$$
\widehat{J}_{k, \beta}(j)=\frac{1}{k} \sum_{i=1}^{k} Y_{\beta}(i, j) / \sum_{i=1}^{k} \tilde{Y}_{\beta}(i, j),
$$

where

$$
\begin{aligned}
& Y_{\beta}(i, j)=\sum_{n=0}^{N(i)} \sum_{\gamma=1}^{\eta_{n}} h_{j}\left(X_{n}^{\gamma}(i)\right) / \beta\left(X_{n}^{\gamma}(i)\right), \\
& \tilde{Y}_{\beta}(i, j)=Y_{\beta}(i, j) \quad \text { with } \quad h_{i}(x) \equiv 1 .
\end{aligned}
$$

Note that when $\beta(x) \equiv 1$ the process reduces to direct simulation of the chain $\left\{X_{n}\right\}$, and the estimators
$\widehat{J}_{i, \beta}$ become the known ratio estimators of the regeneration method (see Crane, Iglehart, 1974).

In the next section we will show that these estimators are asymptotically unbiased estimators and we will introduce a representation for the variancecovariance matrix.

\section{EXPRESSION FOR COVARIANCES}

Set

$$
\begin{aligned}
D(\beta) & =\left(\lim _{k \rightarrow \infty} k \operatorname{Cov}\left(\widehat{J}_{k, \beta}(i), \widehat{J}_{k, \beta}(j)\right)\right)_{i, j=1}^{l}, \\
T(\beta) & =\lim _{k \rightarrow \infty} \sum_{i=1}^{k} T_{i, \beta} / k .
\end{aligned}
$$

By virtue of Law of Large Numbers, $T(\beta)$ is the expectation of the number of steps for all chains in one loop.

Set

$$
\hat{h}_{j}(x)=\widehat{J}_{k, \beta}(j), \quad j=1,2, \ldots, l,
$$

under the condition that all paths begin at $x\left(X_{0}^{1}(i)=\right.$ $x, \quad i=1, \ldots, k)$.

Theorem 1. When conditions (a)-(c) are valid for general Markov chains, estimators $\widehat{J}_{k, \beta}(i)$ are asymptotically unbiased and

$$
\begin{aligned}
D(\beta) & =\left(\int \beta^{-1}(x) \pi(d x)\left(d_{i j}(x)+r_{\beta}\right)\right)_{i, j=1}^{l}, \\
T(\beta) & =\int \beta(x) \pi(d x),
\end{aligned}
$$

where $d_{i j}(x)=E \hat{h}_{i}(x) \hat{h}_{j}(x)-E \hat{h}_{i}(x) E \hat{h}_{j}(x), E$ is the expectation operator, and $r_{\beta}$ is a remainder term.

This theorem is an obvious modification of Theorem 3 in Melas (1993). There it can be found the form of the remainder term as well as arguments for its ignoring.

Note that the value of $d_{i j}(x)$ can be estimated with respect to current data of simulation in the standard way on the basis of the formula from Theorem 1 .

A similar result is valid for finite Markov chains.

Theorem 1 permits us to introduce optimality criteria for the choice of the branching measure. To simplify the notation let us consider that $\pi(d x)=$ $\pi(x) d x$. Set

$$
\tau(x)=\pi(x) \beta(x) / \int \beta(x) \pi(x) d x
$$

$$
\tau_{x}=\pi_{x} \beta_{x} / \sum_{x} \beta_{x} \pi_{x} \quad \text { (finite chains). }
$$


Then $T(\tau)=1$ for any $\beta$. Dropping the remainder term, we obtain the matrix

$$
\begin{aligned}
& \bar{D}(\tau)=\left(\int \tau^{-1}(x) d_{i j}(x) \pi^{2}(x) d x\right) \\
& \bar{D}(\tau)=\left(\sum_{x=0}^{m} \tau_{x}^{-1} \pi_{x}^{2} d_{i j}(x)\right) \quad \text { (finite chains) }
\end{aligned}
$$

As an estimation accuracy criterion let us take quantities

$$
\begin{aligned}
& \operatorname{tr} A \bar{D}(\tau), \\
& \operatorname{det} \bar{D}(\tau)
\end{aligned}
$$

where $A$ is an arbitrary nonnegative-definite matrix. Note that when $A=I$ (where $I$ is identity matrix) and $l=1, \operatorname{tr} A \bar{D}(\tau)$ is just the variance of the estimator $\widehat{J}_{1}$. The statistical meaning of criterion (4) is well known from the theory of regression experimental design (see Kiefer, 1974).

The probability measure $\tau(x)$ is called a design of the simulation experiment.

\section{OPTIMAL EXPERIMENTAL DESIGNS}

Consider the minimization problem for criteria (3) and (4) in the class of probability measures $\tau$ induced by branching densities. The optimal design for criterion (3) can be found with the help of the Schwarz inequality. The direct application of this inequality brings the following result.

Theorem 2. Let the hypothesis of Theorem 1 be satisfied. Then the experimental design minimizing the value of $\operatorname{tr} A D(\tau)$ is given by formula (1), where

$$
\beta(x)=\sqrt{\operatorname{tr} A D(\tau)} .
$$

Let us formulate the results for criterion (4) for finite Markov chains and the case of $h_{i}(x)=\delta_{i x}$, where $\delta_{i x}$ is the Kronecker delta, $i=1,2, \ldots, m$. The general case can be formulated in the same way.

Theorem 3. For finite Markov chains satisfying the conditions $(d)$ and (e), and functions $h_{i}(x)$ of the form $h_{i}(x)=\delta_{i x}, i=1, \ldots, m$, there exists an experiment design minimizing the value of (4). This design is unique and satisfies the relation

$$
\tau(x)=\left(\operatorname{tr} B_{x} B^{-1}\left(\tau^{*}\right)\right)^{1 / 2} \pi_{x} / \sqrt{m},
$$

where

$$
\begin{aligned}
B_{x} & =\left(p_{x y} \delta_{y z}-p_{x y} p_{x z}\right)_{y, z=1}^{m}, \\
x & =0,1, \ldots, m, \\
B(\tau) & =\sum_{x=0}^{m}\left(\pi_{x}^{2} / \tau_{x}\right) B_{x} .
\end{aligned}
$$

The following iterative method can be used to find the optimal design.

Set

$$
\begin{aligned}
& \tau_{0}(y)=\pi_{y}, \quad y=0, \ldots, m \\
& \tau_{k+1}(y, \alpha)=(1-\alpha) \tau_{k}(y)+\alpha \tau_{(x)}(y) \\
& k=0,1,2, \ldots
\end{aligned}
$$

where

$$
\begin{gathered}
\tau_{(x)}(y)=\delta_{x y} \\
x=\arg \max _{x} \frac{\pi_{x}^{2}}{\tau_{k}^{2}(x)} \operatorname{tr} B_{x} B^{-1}\left(\tau_{k}\right) .
\end{gathered}
$$

Set

$$
\begin{gathered}
\alpha_{k}=\arg \min _{\alpha \in[0,1]} \operatorname{det} B\left(\left\{\tau_{k+1}(y, \alpha)\right\}\right), \\
\tau_{k+1}(y)=\tau_{k+1}\left(y, \alpha_{k}\right), \quad y=0,1, \ldots, m .
\end{gathered}
$$

Theorem 4. Under the hypothesis of Theorem 3 for $k \rightarrow \infty$

$$
\operatorname{det} \bar{D}\left(\tau_{k}\right) \rightarrow \operatorname{det} \bar{D}\left(\tau^{*}\right)
$$

where $\operatorname{det} D\left(\tau^{*}\right)=\min \operatorname{det} D(\tau)$.

When applying the branching method in practice, statistical estimators of $\pi_{x}$ with respect to simulation results are to be used instead of the quantities $\pi_{x}$ in the present algorithm. Proofs of theorems 2-4 can be found in Ermakov, Melas (1995).

\section{BRANCHING EFFICIENCY}

Let us study the efficiency of the branching technique for the case of finite Markov chains and the random walk processes.

\subsection{Finite Markov Chains}

Let us investigate the efficiency of the branching method using optimally chosen design $\tau$, with respect to direct simulation, which corresponds to the experiment design $\tau=\pi$. The relative efficiency is defined by the formula

$$
R=\frac{\operatorname{det} \bar{D}\left(\tau^{*}\right)}{\operatorname{det} \bar{D}(\pi)} .
$$

This value depends on the transition matrix $P$, so we write $R=R(P)$.

It is not difficult to verify that $R=1$ in the case when $\pi=(1 /(m+1), \ldots, 1 /(m+1))$, and in the case when the Markov chain turns into a sequence of independent random variables. However, these cases are of no practical interest. The following result demonstrates that the relative efficiency can be arbitrarily large. 
Theorem 5. Under the hypothesis of Theorem 3 when $m=1$

$$
\sup _{P: \pi P=\pi} R(P)=\min \left\{\frac{1}{1+2 \sqrt{\pi_{0} \pi_{1}}}, \max \left(\pi_{0}, \pi_{1}\right)\right\},
$$

when $m>1$

$$
\sup _{P} R(P)=\infty \text {. }
$$

The proof of Theorem 5 can be found in Ermakov, Melas (1995). The branching technique's efficiency for the special class of chains for which $p_{i i+l}=0$, $l>1$ is investigated in Melas (1994). And a gener-

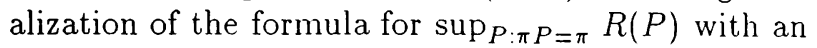
arbitrary given $\pi$ and arbitrary $m$ was developed in Melas, Fomina (1997).

\subsection{Random Walk}

Consider a random walk process on a line

$$
S_{1}=0, \quad S_{n+1}=S_{n}+X_{n}, \quad k=1,2, \ldots,
$$

where $X_{n}, k=1,2, \ldots$ are independent random variables with common density function $f(x)$, and connect it with the waiting process

$$
W_{1}=0, \quad W_{n+1}=\max \left(0, W_{n}+K_{n}\right), \quad n=1,2, \ldots
$$

It is known (see Feller, 1970) that the quantities $W_{n}$ and

$$
M_{n}=\max \left\{S_{1}, \ldots, S_{n}\right\}
$$

have the same distribution. Under an additional condition on $E X_{1}, M_{n} \rightarrow M$ and $W_{n} \rightarrow W$ in distribution, where $M$ and $W$ are random variables.

Set $\theta=P\{W \geq v\}=P\{M \geq v\}$. This value cannot be calculated analytically and the problem is the estimation of $\theta$ with the help of simulation. This problem has a number of applications. It had been investigated in Siegmund (1976) and Asmussen (1985).

Set $\pi(0)=P\{W=0\}, \pi(x)=W^{\prime}(x), W(x)=$ $P\{W<x\}, \pi(x)=0$ for $x<0, X_{n}=W_{n}, S=\{0\}$, $\pi(d x)=a f(x) d x, a=1 /(1-F(0))$, and

$$
\begin{gathered}
1-F(0)=\int_{0}^{\infty} f(t) d t \\
h_{1}(x)=0, \quad x<v, \quad h_{1}(x)=1, \quad x \geq v, l=1 .
\end{gathered}
$$

Then it is evident that conditions (a), (b) and (c) are valid. The problem is the estimation of the functional

$$
J=J_{1}=\int h_{1}(x) \pi(x) d x .
$$

Suppose that $f(x)$ satisfies the condition

$$
\int_{-\infty}^{\infty} e^{\lambda_{0} t} f(t) d t=0
$$

for some positive $\lambda_{0}$. Set $\beta_{0}(x) \equiv 0, \beta^{*}(t)=e^{\lambda_{0} t}$, $0<t<v, \beta^{*}(t)=e^{\lambda_{0} t}, t \geq v$. Define the relative efficiency by the formula

$$
R=R_{v}=\frac{T_{\beta} \cdot D \widehat{J}_{\beta} \cdot}{T_{\beta_{0}} D \widehat{J}_{\beta_{0}}} .
$$

It is known (see Feller, 1970) that as $v \rightarrow \infty \theta \sim$ $e^{-\lambda_{0} t} \rightarrow 0$.

Theorem 6. Let the conditions formulated above be satisfied and for some $\varepsilon>0$

$$
\int_{-\infty}^{\infty} e^{2\left(\lambda_{0}+\varepsilon\right) t} f(t) d t<\infty
$$

Then for $v \rightarrow \infty$

$$
R_{v}=O\left(\frac{1}{\theta \ln ^{2}(1 / \theta)}\right), \quad \theta \sim e^{-\lambda_{0} v} .
$$

Thus the relative efficiency converges to $\infty$ as $v \rightarrow$ $\infty$. Theorem 6 is a modification of Theorem 5.5 from Ermakov, Melas (1995), chapter 4.

Since Theorem 6 describes only the asymptotic behavior of the relative efficiency we give numerical results in the next section.

\section{NUMERICAL EXAMPLE}

Let us consider a queueing system of the type $G I / G / 1 / \infty$. This system can be described in the following manner. Demands come in moment $t_{1}, t_{2}, \ldots$ and need service time $u_{1}, u_{2}, \ldots$, respectively. Demands are served in order of coming. We assume that $\left\{v_{i}=t_{i+1}-t_{i}\right\}$ and $\left\{u_{i}\right\}$ are independent random variables. In the simulation experiments it is assumed that $v_{i}$ and $u_{i}(i=1,2, \ldots)$ have exponential distribution with parameters $a$ and 1, respectively. The problem is to estimate the steady-state probability $\theta=\lim _{n \rightarrow \infty} P\left\{W_{n}<v\right\}$, where $W_{n}$ is the waiting time for $n$-th demand and $v$ is a given magnitude.

To compare the branching technique (BT) and the usual regeneration technique (RT) we simulated the corresponding random walk by both methods during 100,000 loops. The magnitude $v$ was chosen so that $\theta=10^{-2}, 10^{-3}, \ldots, 10^{-6}, a=1 / 2$. Denote by $I$ the magnitude

$$
I=\theta^{2} /(E T D \hat{\theta})
$$

where $\theta$ is the proper value of the estimated parameters, ET is the mean length of the simulated paths and $D \widehat{\theta}$ is the sample variance.

We have obtained results presented in the following table:

$\begin{array}{cccccc}\ln (1 / \theta) & 2 & 3 & 4 & 5 & 6 \\ I(R T) & 0,385 & 0,026 & 0,000 & 0,000 & 0,000 \\ I(B T) & 0,686 & 0,298 & 0,185 & 0,083 & 0,041\end{array}$


The table shows that for $\theta \leq 10^{-3}$ the relative efficiency is significant.

\section{ACKNOWLEDGMENTS}

This work was partly supported by Russian Foundation of Basic Research (N 96-01-00644).

\section{REFERENCES}

Asmussen, S. 1985. Conjugate process and the simulation of ruin problems. Stochastic Process Applications 20 (2): 213-229.

Bratley, P., B. L. Fox, and L. E. Schrage. 1987. A guide to simulation. 2nd ed. New York: SpringerVerlag,

Cheng, R. C. H., and W. Holland. 1995. The effect of input parameters on the variability of simulation output. Proceedings of the Second United Kingdom Simulation Society Conference (ed. R.C.H.Cheng and R.J.Pooley) North Berwick, April Edinburg University, 29-36.

Crane, A. M., and D. L. Iglehart. 1974. Simulating stable stochastic systems. 2: Markov chains. Journal of Association of Computing Machinery 21 (1): 114-123.

Ermakov, S. M., and V. B. Melas. 1995. Design and analysis of simulation experiments. Dordrecht, London: Kluwer Academic Publishers.

Feller, W. 1970. An introduction to probability theory and its applications, vol. 2. 2nd ed. New York: Wiley.

Glasserman, P., P. Heidelberger, P. Shahabuddin, and T. Zajic. 1996. Splitting for rare simulation analysis of simple cases. In Proceedings of the 1996 Winter Simulation Conference, IEEE: Computer Society Press, 302-308.

Glynn, P. W., and D. L. Iglehart. 1988. Simulation method for queues. Queueing Systems 3 (2): 221256.

Kahn, H. 1956. Use of different Monte Carlo sampling techniques. Symposium on Monte Carlo methods, ed. H. A. Meyer. New York: John Wiley and Sons, 146-190.

Kiefer, J. 1974. General equivalence theory for optimum designs. Annals of Mathemathical Statistics 30: 271-294.

Kiefer, J., and J. Wolfowitz. 1959. Optimum designs in regression problems. Annals of Mathematical Statistics 30 (2): 271-294.

Melas, V. B. 1993. Optimal simulation design by branching technique. Model Oriented Data Analysis, ed. W. G. Muller, H. P. Wynn, and A. A., Zhigljavsky. Heidelberg: Physica-Verlag, 113-127.
Melas, V. B. 1994. Branching technique for Markov chains simulation (finite state case). Statistics 25 (2): 159-171.

Melas, V. B. 1995. Efficiency of Branching Technique for Markov Chain Simulation. Proceedings of the 1995 Summer Computer Simulation Conference, ed. T.I. Oren, and L.G. Birta, Ottawa, SCS, 108111.

Melas, V. B., and T. V. Fomina. 1997. On the efficiency of the branching technique for finite Markov chains. Vestnik of St. Petersburg State University, ser. 1 (8): 28-33. (Russian).

Nummelin, E. 1984. General irreducible Markov chains and non-negative operators. Cambridge: Cambridge University Press.

Siegmund, D. 1976. Importance sampling in the Monte Carlo study of sequential tests. Annals of Statistics 4 (4): 673-684.

Sigman, K. 1988. Queues as Harris recurrent Markov chains. Queueing systems 3: 179-198.

\section{AUTHOR BIOGRAPHY}

VIATCHESLAV B. MELAS is a professor in the Department of Mathematics and Mechanics at St. Petersburg State University. He received an M.S. degree in mathematics from Leningrad University in 1971, and he received a Ph.D. degree in theory of probability and mathematical statistics and a Doctor of Science degree in numerical mathematics and theory of probability from Leningrad University in 1977 and 1994 respectively. His research interests concentrate in stochastic simulation, especially in variance reduction techniques, and in optimal design theory. 